Check for updates

Cite this: RSC Adv., 2021, 11, 13571

Received 19th February 2021

Accepted 16th March 2021

DOI: $10.1039 / \mathrm{d} 1 \mathrm{ra01348c}$

rsc.li/rsc-advances

\section{Bromination and increasing the molecular conjugation length of the non-fullerene small- molecule acceptor based on benzotriazole for efficient organic photovoltaics $\dagger$}

\author{
Na Zhang, Zhe Li, Can Zhu, (DD Hongjian Peng (DD * and Yingping Zou \\ Two novel non-fullerene acceptors, namely $\mathrm{BZIC}-2 \mathrm{Br}$ and $\mathrm{Y9}-2 \mathrm{Br}$, were synthesized by employing \\ a ladder-type electron-deficient-based fused ring central with a benzotriazole core. $\mathrm{Y} 9-2 \mathrm{Br}$ is obtained \\ by extending the conjugate length of BZIC-2Br. Compared with BZIC-2Br, Y9-2Br possesses a lower \\ optical bandgap of $1.32 \mathrm{eV}$ with an absorption edge of $937 \mathrm{~nm}$, exhibiting broader and stronger \\ absorption band from 600 to $900 \mathrm{~nm}$. Moreover, $Y 9-2 \mathrm{Br}$ exhibits excellent photovoltaic properties with \\ $V_{\text {oc }}$ of $0.84 \mathrm{~V}, J_{\text {sc }}$ of $21.38 \mathrm{~mA} \mathrm{~cm}$ c $^{-2}$ and FF of $67.11 \%$, which achieves an impressive PCE of $12.05 \%$. Our \\ study demonstrates that bromination and effective extension of the conjugate length can modulate \\ performance from different aspects to optimize photovoltaic characteristics.
}

\section{Introduction}

The photoactive layers of bulk-heterojunction (BHJ) organic solar cells (OSCs) are usually constructed with electron donors and acceptors. ${ }^{\mathbf{1}}$ Electron acceptors include commonly fullerenebased acceptors or non-fullerene fused-ring acceptors (NFAs). Over the past two decades, great efforts have been made to improve the power conversion efficiency (PCE) by over $18 \%$ for single-junction devices. ${ }^{2-5}$ In general, the performance of OSCs can be evaluated by the following three main parameters: the open circuit voltage $\left(V_{\mathrm{oc}}\right)$, short-circuit current density $\left(J_{\mathrm{sc}}\right)$ and fill factor (FF). Among acceptor innovations, NFAs have become the main materials of OSCs because of reduced energy loss for a high $V_{\mathrm{oc}}$ and extended absorption range for a high $J_{\mathrm{sc}}$, which is essential requirements for high PCE., ${ }^{6,7}$ The first breakthrough was made in 2015 for the development of NFAs. Zhan et al. reported a fused-ring electron acceptor (FREA), named ITIC, with an acceptor-donor-acceptor (A-D-A) structure. ${ }^{3}$ The structure was further optimized to boost the PCE of OSCs to $13-15 \%{ }^{8-12}$ In order to further improve the photoelectric properties of A-D-A type NFAs, an electron-deficient $\left(\mathrm{A}^{\prime}\right)$ unit was introduced into the central fused-ring of the A-D-A molecule to form an A$\mathrm{DA}^{\prime} \mathrm{D}-\mathrm{A}$ structure by Zou et al., named BZIC ${ }^{2}$ (Fig. 1), based on a ladder-type thieno[3,2- $b]$ pyrrolo-fused pentacyclic benzotriazole (BZTP) as the core and end-capped with 1,1dicyanomethylene-3-indanone (INCN), ${ }^{13-15}$ which exhibits an

College of Chemistry and Chemical Engineering, Central South University, Changsha, Hunan 410083, China. E-mail: hongjianpeng@126.com; Tel: +86-731-88879616 $\dagger$ Electronic supplementary information (ESI) available: The figures of ${ }^{1} \mathrm{H}$ NMR for synthesized compounds, mass spectrum and thermogravimetric analysis. See DOI: $10.1039 / \mathrm{d} 1 \mathrm{ra01348 \textrm {c }}$ excellent PCE of $6.3 \%$ with $V_{\text {oc }}$ of $0.84 \mathrm{~V}, J_{\mathrm{sc}}$ of $12.67 \mathrm{~mA} \mathrm{~cm}^{-2}$ and $\mathrm{FF}$ of $59 \%$. The emergence of $\mathrm{Y} 6$ in 2019 broke the record for the highest OSCs at that time, with PCE exceeding 15\%. On this basis, the subsequent development of Y series NFAs is constantly updated, promoting the progresses of OSCs with the high PCE ranging of $15-18 \% .^{5,16,17}$ Compared to ITIC-series FREAs, the main difference of Y-series NFAs is the introduction of the electron-deficient core. Besides, the pyrrole ring is also used to replace cyclopentadiene. ${ }^{\mathbf{1} 18}$ The A-DA'D-A-type molecules possess typical structural superiority. First, in order to strengthen D-A interactions and enhance intermolecular and intramolecular interactions, the electron-deficient unit, such as benzothiadiazole (BT), was introduced ${ }^{\mathbf{1 9}}$ into the fused ring, contributing to improve the electron mobility and molecular aggregation. ${ }^{20}$ For the conjugated system, pyrrole-bridging rings can be used as electron donors due to their strong electrondonating ability, ${ }^{21}$ which is beneficial to upshift the highest occupied molecular orbital (HOMO) energy level and reduce the bandgap of NFAs. Finally, there are two nitrogen atoms in the two pyrrole units, which link to the alkyl chains ${ }^{22}$ to suppress severe aggregation due to their steric hindrance and improve the solubility of NFAs. The typical A-DA'D-A structure makes for a low nonradiative recombination loss from electroluminescence quantum efficiency based on these acceptors. ${ }^{23,24}$

Therefore, the structure optimization of Y-series NFAs generally starts from the following three aspects: (1) core engineering influencing the energy levels to regulate the performance of devices. ${ }^{25,26}$ For example, Y1, with benzotriazole (BT) as an electron-deficient core, showed the strong absorption with a narrow bandgap of $1.44 \mathrm{eV}^{27}$ To improve the photovoltaic performance further, BT was replaced by benzothiadiazole 


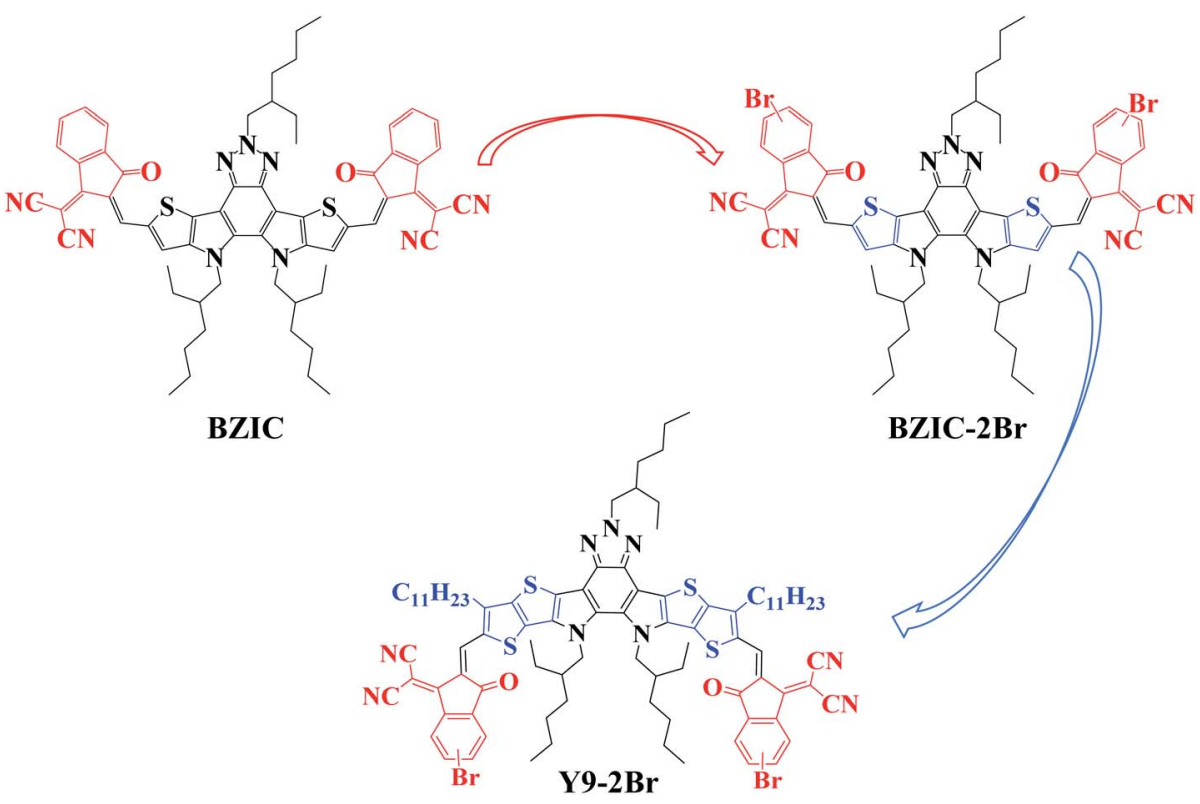

Fig. 1 The chemical structures of BZIC, BZIC-2Br and Y9-2Br.

(BTA) to obtain Y5, which showed the deeper HOMO level and the narrower bandgap of $1.38 \mathrm{eV}$, when compared with $\mathrm{Y}_{1} ;^{28}(2)$ alkyl chain engineering to improve the solubility of materials and induce steric hindrance effects to minimize the energetic disorder. ${ }^{16,24,29}$ For instance, Hou et al. designed a novel molecule named BTP-4F-12, which was obtained by changing 2ethylhexyl on Y6 to 2-butyloctyl. BTP-4F-12 not only showed better solubility but also tighter $\pi-\pi$ stacking, which resulted in an improved PCE of $16.4 \%$, which is superior to that of Y6 $(15.7 \%) ;{ }^{30}(3)$ terminal engineering can be used to adjust energy levels, absorption, blend morphology and charge dynamics. ${ }^{31}$ Chen et al. synthesized an NFA, BTP-M, by one methyl group instead of fluorine atoms on the IC terminal, showing higher energy levels and blending with PBDB-T, OSCs with a PCE of $14.24 \%{ }^{32}$

Halogens have been widely used in organic semiconductors. $^{33-35}$ The introduction of halogen atoms can adjust the photoelectric properties of materials, such as the absorption and energy levels. ${ }^{36-42}$ Therefore, for non-fullerene OSCs, halogenation is a very promising molecular design strategy to further improve the performance. ${ }^{43}$ Compared to the fluorination and chlorination, the bromine atom is rarely incorporated into the non-fullerene acceptors. In 2017, Li et al. explored the bromination of $\mathrm{NFAs}^{44}$ and then Chen et al. found that bromine atoms were introduced into NFAs, showing the same or even better device performance in comparison with $\mathrm{F}$ and $\mathrm{Cl}^{45} \mathrm{Zhang}$ further studied the effects of introducing one or two bromine atoms into NFAs, and the results show that one brominesubstituted molecule exhibits better device performance. ${ }^{46}$ Therefore, when the brominated small molecules are used as acceptors, the device performances may be further improved. ${ }^{47-50}$ In addition, increasing the molecular conjugation length of molecules is another measure to optimize photovoltaic properties. ${ }^{51-56}$ The first reported A-DA'D-A NFA,
BZIC, showed a moderate PCE of $6.3 \% .^{2}$ When the pentacyclic ring was extended to a heptacyclic one to obtain Y1, resulting in PCEs of $13.42 \%$, the reason of which is that increasing the molecular conjugation length can improve light absorption capability and charge transport. ${ }^{28}$

Therefore, based on the above-mentioned strategies, BZIC$2 \mathrm{Br}$ (bromination of BZIC) and $\mathrm{Y9}-2 \mathrm{Br}$ (increasing the molecular conjugation length of BZIC-2Br) were designed and synthesized, respectively (Fig. 1). With the introduction of bromine atom, the absorption spectra of BZIC-2Br and Y9-2Br exhibit red-shift to obtain a narrower optical bandgap. Increasing the molecular conjugation length of molecules can regulate the HOMO and the lowest unoccupied molecular orbital (LUMO) energy levels, enhance the light-harvesting capability of molecules, broaden the absorption range, reduce the bandgap and enhance charge mobility. Finally, Y9-2Br exhibits the best excellent performance compared with BZIC and BZIC-2Br.

\section{Results and discussion}

\subsection{Synthesis and characterization}

As shown in Scheme S1 (ESI), $\dagger$ BZIC-2Br and Y9-2Br were synthesized via Knoevenagel condensation reactions between the corresponding dialdehyde and end group (2-(6-bromo-3oxo-2,3-dihydro- $1 \mathrm{H}$-inden-1-ylidene)malononitrile (INCN-Br)), respectively. The chemical structures of these new small molecule acceptors (SMAs) were fully characterized by ${ }^{1} \mathrm{H}$ nuclear magnetic resonance (NMR). The molecular mass was confirmed using an Autoflex III matrix-assisted laser desorption ionization mass spectrometer (MALDI-TOF-MS) (Fig. S11†). Thermogravimetric analysis (TGA) was conducted at a heating rate of $10{ }^{\circ} \mathrm{C} \min ^{-1}$ under nitrogen, as shown in Fig. S12, $\dagger$ which indicates that BZIC-2Br and $\mathrm{Y9}-2 \mathrm{Br}$ have good thermal stability 
with decomposition temperatures at $310{ }^{\circ} \mathrm{C}$ and $318{ }^{\circ} \mathrm{C}$, meeting the requirements of device fabrication. BZIC-2Br and Y9-2Br show good solubility in commonly used solvents, including chloroform (CF) and dichloromethane (DCM), particularly greater than $20 \mathrm{mg} \mathrm{mL} \mathrm{mL}^{-1}$ in chloroform at room temperature.

\subsection{Photoelectric properties}

2.2.1 Electrochemical properties. The HOMO energy level and LUMO energy level of BZIC, ${ }^{2}$ BZIC-2Br and $\mathrm{Y9}-2 \mathrm{Br}$ were measured via the cyclic voltammetry (CV) method. As shown in Fig. 2(a), the initial reduction/oxidation potentials of BZIC, BZIC-2Br and $\mathrm{Y9}-2 \mathrm{Br}$ are $-0.48 / 1.06,-0.51 / 1.45$ and -0.73 / $1.17 \mathrm{~V}$, respectively. Thus, according to the equation $E_{\mathrm{HOMO}} /$ $E_{\mathrm{LUMO}}=-e\left(\varphi_{\mathrm{ox}} / \varphi_{\text {red }}+4.41\right)$, the HOMO/LUMO energy levels of BZIC, BZIC-2Br and $\mathrm{Y9}-2 \mathrm{Br}$ are $-5.42 /-3.88,-5.85 /-3.92$, $-5.53 /-3.64 \mathrm{eV}$, respectively. In addition, the electrochemical bandgaps are $1.54 \mathrm{eV}, 1.93 \mathrm{eV}$ and $1.89 \mathrm{eV}\left(E_{\mathrm{g}}^{\mathrm{CV}}=E_{\mathrm{LUMO}}-\right.$ $\left.E_{\text {Hомо }}\right)$, respectively. Compared with BZIC, the LUMO level of BZIC-2Br shows a downward trend, resulting from the electronwithdrawing effects of bromine atoms. ${ }^{57}$ And thieno[3,2- $\left.b\right]$ thiophene instead of a thiophene segment of the molecules can extend the fused central ring, which elevates the LUMO energy levels. The higher LUMO energy level of the acceptor is beneficial for the OSC device to obtain a higher $V_{\text {oc }}$.

2.2.2 Optical properties. Fig. 2(b) shows the ultraviolet visible (UV-Vis) absorption spectra of the small molecule acceptors BZIC-2Br and $\mathrm{Y} 9-2 \mathrm{Br}$ in the chloroform solution and thin film. The relevant optical data are listed in the Table 1. From the spectra, it can be seen that BZIC-2Br and Y9-2Br have
Table 1 Photoelectric data of $\mathrm{BZIC}-2 \mathrm{Br}$ and $\mathrm{Y} 9-2 \mathrm{Br}$ in the film state

\begin{tabular}{lcccccc}
\hline & $\begin{array}{c}\lambda_{\max } \\
\text { Parameter } \\
(\mathrm{nm})\end{array}$ & $\begin{array}{c}\lambda_{\text {edge }} \\
(\mathrm{nm})\end{array}$ & $E_{\mathrm{g}}^{\mathrm{opt}}(\mathrm{eV})$ & $E_{\text {Hомо }}(\mathrm{eV})$ & $E_{\mathrm{LUMO}}(\mathrm{eV})$ & $E_{\mathrm{g}}^{\mathrm{CV}}(\mathrm{eV})$ \\
\hline BZIC $^{2}$ & 770 & 857 & 1.45 & -5.42 & -3.88 & 1.54 \\
BZIC-2Br & 807 & 886 & 1.40 & -5.85 & -3.92 & 1.93 \\
Y9-2Br & 855 & 937 & 1.32 & -5.53 & -3.64 & 1.89
\end{tabular}

absorptions in a range of 350-940 $\mathrm{nm}$. In particular, the maximum absorption peaks are $807 \mathrm{~nm}$ and $855 \mathrm{~nm}$ in the film, and the edges of absorption are $886 \mathrm{~nm}$ and $937 \mathrm{~nm}$ in the film. Compared with BZIC, BZIC-2Br and Y9-2Br exhibit a significant red-shift, as shown in the absorption spectrum (about 29 and 80 $\mathrm{nm})$ and the narrower optical bandgap $\left(E_{\mathrm{g}}^{\mathrm{opt}}, E_{\mathrm{g}}^{\mathrm{opt}}=1240 / \lambda_{\text {edge }}\right)$ of 1.40 and $1.32 \mathrm{eV}$ in the thin film, which shows that bromination and increasing the molecular conjugation length can enhance the light-harvesting capability and reduce the bandgap.

\subsection{Photovoltaic properties}

To further investigate the photovoltaic performance, poly[(2,6(4,8-bis(5-(2-ethylhexyl)thiophen-2-yl)-benzo[1,2- $\left.b: 4,5-b^{\prime}\right]$ dithiophene))-alt-(5,5-(1', $3^{\prime}$-di-2-thienyl-5', $7^{\prime}$-bis(2-ethyl-hexyl)benzo $\left[1^{\prime}, 2^{\prime}-c: 4^{\prime}, 5^{\prime}-c^{\prime}\right]$ dithiophene-4,8-dione))] (PBDB-T) was chosen as the donor to match with BZIC-2Br and $\mathrm{Y9} 9 \mathrm{Br}$, respectively, used as the active layer to construct OSCs, while the device structure was prepared as ITO/PEDOT:PSS/PBDB-T:BZIC-2Br(PBDB-T:Y92Br)/PDINO/Al (Fig. 2(c)). The current-voltage spectra measured under $\mathrm{AM} 1.5 \mathrm{G}$ illumination $100 \mathrm{~mW} \mathrm{~cm}^{-2}$
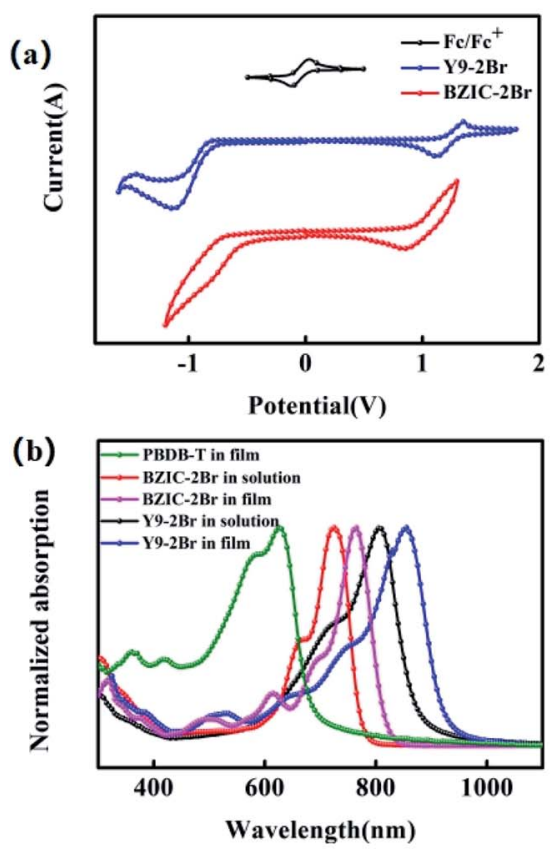

(c)

(d)

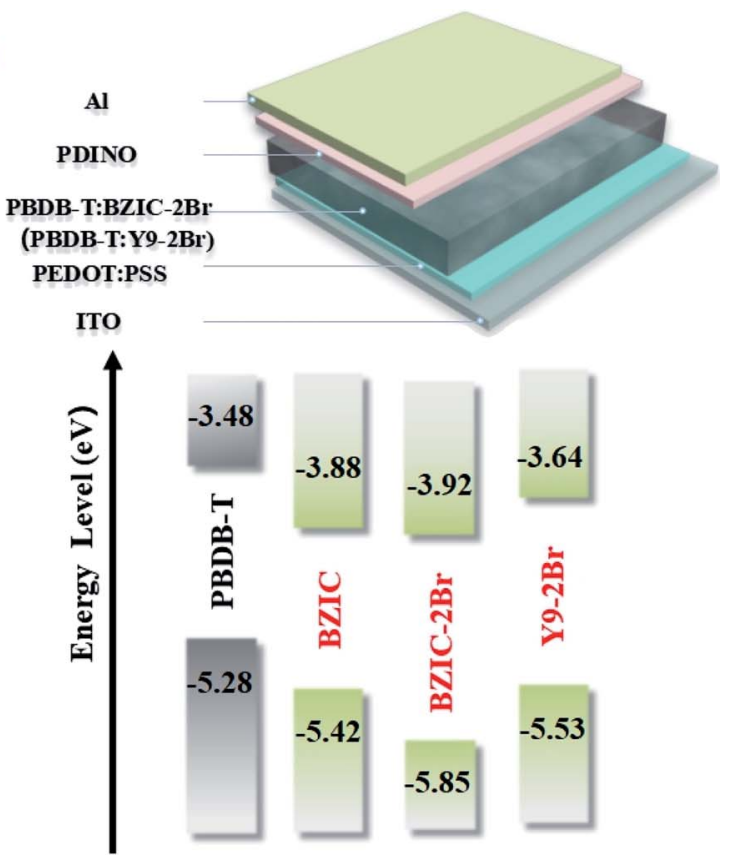

Fig. 2 (a) Cyclic voltammetry curves of BZIC-2Br and $\mathrm{Y9}-2 \mathrm{Br}$; (b) UV-Visible absorption spectra of PBDB-T in film, BZIC-2Br and Y9-2Br in solution and thin film state, respectively; (c) device structure used for OSC fabrication; (d) energy level diagrams of donors (PBDB-T), and acceptors (BZIC, ${ }^{2} \mathrm{BZIC}-2 \mathrm{Br}$ and $\mathrm{Y9}-2 \mathrm{Br}$ ). 
conditions are shown in Fig. 3(a), and the corresponding parameters are listed in Table 2. The optimized OSCs based on BZIC-2Br showed a $J_{\text {sc }}$ of only $15.34 \mathrm{~mA} \mathrm{~cm}^{-2}$ due to poor lightharvesting capability, resulting from weak absorption in the near-infrared region (NIR), and a relatively low PCE of 7.51\%. However, device based on the PBDB-T:Y9-2Br $(1: 1.2 \mathrm{w} / \mathrm{w})$ blend achieved an impressive PCE of $12.05 \%$ with a $V_{\mathrm{oc}}$ of $0.84 \mathrm{~V}, J_{\mathrm{sc}}$ of $21.38 \mathrm{~mA} \mathrm{~cm}^{-2}$ and $\mathrm{FF}$ of $67.11 \%$, under thermal annealing (TA) at $100{ }^{\circ} \mathrm{C}$ and the $0.5 \%$ additive 1 -chloronaphthalene (CN) conditions. Moreover, Fig. 3(b) shows the EQE spectra of the PBDB-T:BZIC-2Br and PBDB-T:Y9-2Br-based optimized device. The EQE of PBDB-T:BZIC-2Br at 300-960 nm reaches 50-65\%, compared with that, PBDB-T:Y9-2Br shows the wider absorption (300-990 nm) and higher EQE (75-85\%), which agrees quite well with the $J_{\mathrm{sc}}$ value from the $J-V$ curve within a $5 \%$ mismatch.

As a result, in order to enhance the light-harvesting capability of molecules and then increase the $J_{\mathrm{sc}}$ of OSCs, it is necessary to enlarge the conjugated length of the molecules. Moreover, the effective extension of the conjugate length can elevate the LUMO energy level (shown in Fig. 2(d)) to obtain the higher $V_{\mathrm{oc}}$ and acquire the higher PCE, eventually.

\subsection{Mobility}

The space-charge limited current (SCLC) method was used to investigate the hole and electron mobilities. The dependences of the square root of current density $\left(J^{1 / 2}-V\right)$ on voltage are displayed in Fig. 3(c) and (d). The SCLC is described as follows. ${ }^{58}$

$$
J_{\mathrm{SCLC}}=\frac{9}{8} \varepsilon_{0} \varepsilon_{\mathrm{r}} \mu \frac{V^{2}}{d^{3}}
$$

( $J$ is the current density, $\mu$ is the hole or electron mobility, $V$ is the internal voltage in the device, $\varepsilon_{\mathrm{r}}$ is the relative dielectric constant of active layer material, $\varepsilon_{0}$ is the permittivity of empty space, and $d$ is the thickness of the active layer.)

For the devices based-on PBDB-T:BZIC-2Br and PBDB-T:Y9$2 \mathrm{Br}$, respectively, the electron mobility $\left(\mu_{\mathrm{e}}\right)$ were $8.90 \times 10^{-6}$ and $2.08 \times 10^{-4} \mathrm{~cm}^{2} \mathrm{~V}^{-1} \mathrm{~s}^{-1}$, and the hole mobility $\left(\mu_{\mathrm{h}}\right)$ were $6.03 \times 10^{-6}$ and $1.86 \times 10^{-4} \mathrm{~cm}^{2} \mathrm{~V}^{-1} \mathrm{~s}^{-1}$. The ratio $\left(\mu_{\mathrm{e}} / \mu_{\mathrm{h}}\right)$ of the PBDB-T:Y9-2Br-based device (1.12) is closer to 1 than that of the PBDB-T:BZIC-2Br-based device (1.48) (as shown in Table 3), which indicates that the carrier mobility of the PBDB-T:Y9-2Brbased device is more balanced than that of the PBDB-T:BZIC$2 \mathrm{Br}$-based device. The results exhibit that the effective extension of the conjugate length is a necessary measure to enhance the intermolecular charge transport and charge transfer efficiency.

\subsection{Morphology}

The morphology of OSCs plays a key role in photovoltaic properties. Good miscibility is desirable for maximum exciton separation and transport. To evaluate the effects of the microstructure of active layers on device performance, the morphologies of PBDB-T:BZIC-2Br and PBDB-T:Y9-2Br blend films were investigated by atomic force microscopy (AFM). As is shown in Fig. 4(a) and (c), the root-mean-square $\left(R_{\mathrm{q}}\right)$ surface roughness value for the blend films of PBDB-T:BZIC-2Br and PBDB-T:Y9$2 \mathrm{Br}$ are $2.22 \mathrm{~nm}$ and $1.68 \mathrm{~nm}$, respectively. The blend film of PBDB-T:Y9-2Br exhibits the smooth morphology with smaller $R_{\mathrm{q}}$, which can also is observed in TEM (Fig. 4(b) and (d)). These morphological features may lead to the remarkably high $J_{\mathrm{sc}}$ and FF of the devices.
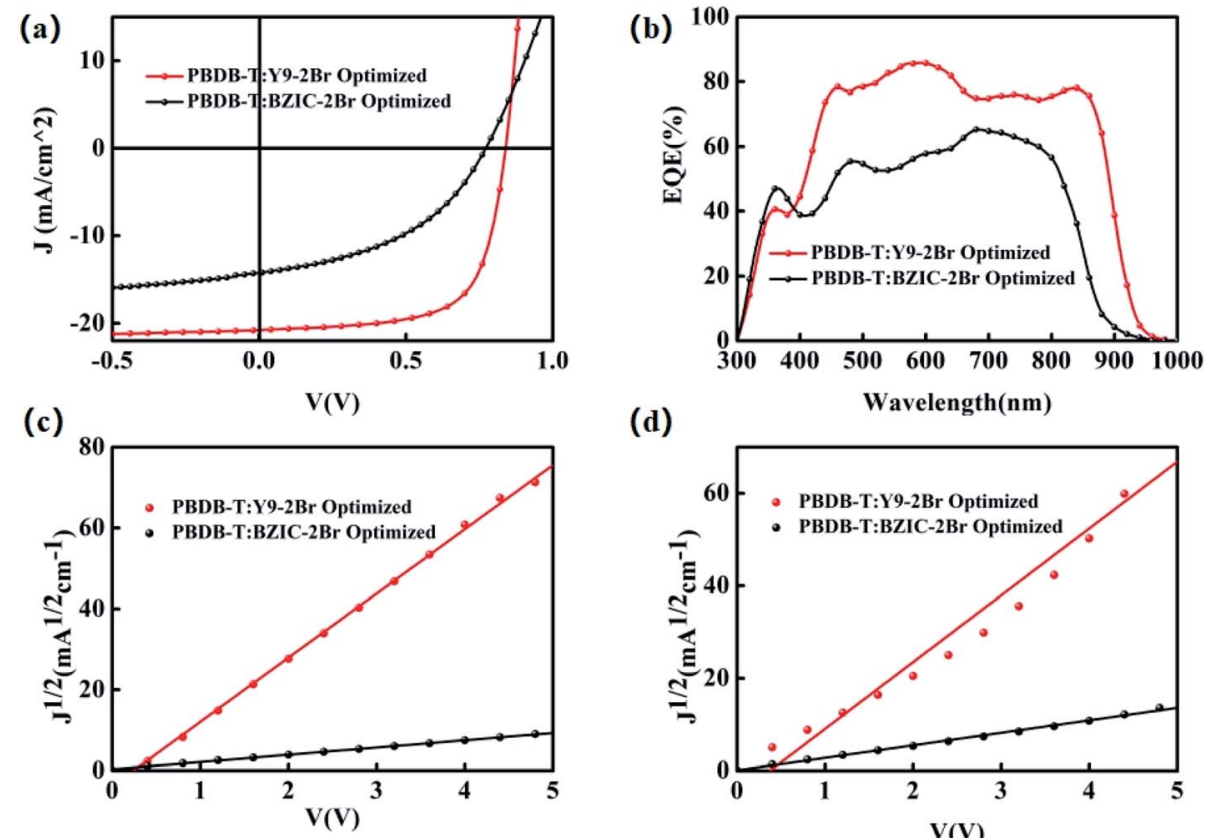

(d)

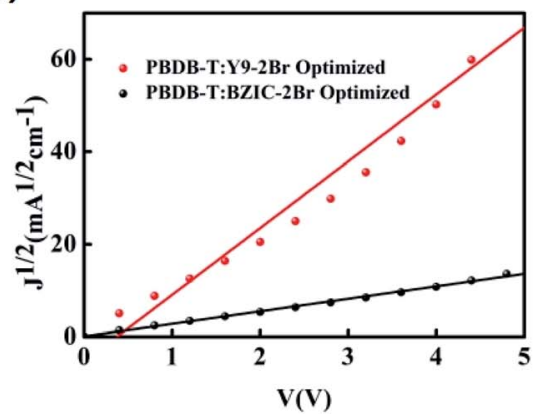

Fig. 3 (a) The J-V curves, (b) the EQE spectra, (c) the electron mobilities and (d) the hole mobilities of the optimal OSCs based on PBDB-T:BZIC$2 \mathrm{Br}$ and $\mathrm{PBDB}-\mathrm{T}: \mathrm{Y} 9-2 \mathrm{Br}$, respectively. 
Table 2 The photovoltaic parameters of devices based on PBDB-T:BZIC-2Br and PBDB-T:Y9-2Br

\begin{tabular}{|c|c|c|c|c|c|c|c|}
\hline Acceptor & $\mathrm{D}: \mathrm{A}(\mathrm{w} / \mathrm{w})$ & Additive, vol\% & $\begin{array}{l}\text { Annealing, } \\
{ }^{\circ} \mathrm{C}\end{array}$ & $V_{\mathrm{oc}}, \mathrm{V}$ & $J_{\mathrm{sc}}, \mathrm{mA} \mathrm{cm}^{-2}$ & $\mathrm{FF}, \%$ & PCE, \% \\
\hline BZIC-2Br & $1: 1.2$ & $\mathrm{CN}(0.5)$ & 100 & 0.75 & 15.34 & 65.19 & $7.51(7.21 \pm 0.3)$ \\
\hline $\mathrm{Y9}-2 \mathrm{Br}$ & & & & 0.84 & 21.38 & 67.11 & $12.05(11.75 \pm 0.3)$ \\
\hline
\end{tabular}

Table 3 The electron-only mobility and hole-only mobility of devices based-on PBDB-T:BZIC-2Br and PBDB-T:Y9-2Br

\begin{tabular}{llll}
\hline & $\mu_{\mathrm{e}}\left(\mathrm{cm}^{2} \mathrm{~V}^{-1} \mathrm{~s}^{-1}\right)$ & $\mu_{\mathrm{h}}\left(\mathrm{cm}^{2} \mathrm{~V}^{-1} \mathrm{~s}^{-1}\right)$ & $\mu_{\mathrm{e}} / \mu_{\mathrm{h}}$ \\
\hline BZIC-2Br & $8.90 \times 10^{-6}$ & $6.03 \times 10^{-6}$ & 1.48 \\
Y9-2Br & $2.08 \times 10^{-4}$ & $1.86 \times 10^{-4}$ & 1.12
\end{tabular}

\section{Conclusions}

In summary, two non-fullerene acceptors using the A-DA'D-A structure multifused benzotriazole central core are designed and synthesized. Compared with BZIC, the absorption spectrum of BZIC-2Br exhibits red-shift due to the narrower optical bandgap. By using a medium bandgap polymer PBDB-T as the electron donor, OSCs based on PBDB-T:BZIC-2Br and PBDBT:Y9-2Br exhibit different properties. Compared with BZIC2Br, PBDB-T:Y9-2Br exhibits the more outstanding performance. The obvious red-shift (about $51 \mathrm{~nm}$ ) of the absorption spectrum and LUMO upshift of Y9-2Br in the thin film can simultaneously increase $V_{\mathrm{oc}}$ and $J_{\mathrm{sc}}$, and good morphological can acquire high FF. The results demonstrate that the introduction of bromine can enhance the lightharvesting capability and reduce the bandgap, and effective extension of the conjugate length can regulate the HOMO and LUMO energy levels to decrease energy levels, enhance the lightharvesting capability of molecules, increase the absorption and reduce the bandgap and enhance charge mobility. These changes will in turn contribute to the remarkably high $V_{\text {oc }}$ of $0.84 \mathrm{~V}, J_{\mathrm{sc}}$ of $21.38 \mathrm{~mA} \mathrm{~cm}^{-2}$ and an FF of $67.11 \%$, and PCE of $12.05 \%$ achieved by the devices.

\section{Conflicts of interest}

There are no conflicts to declare.
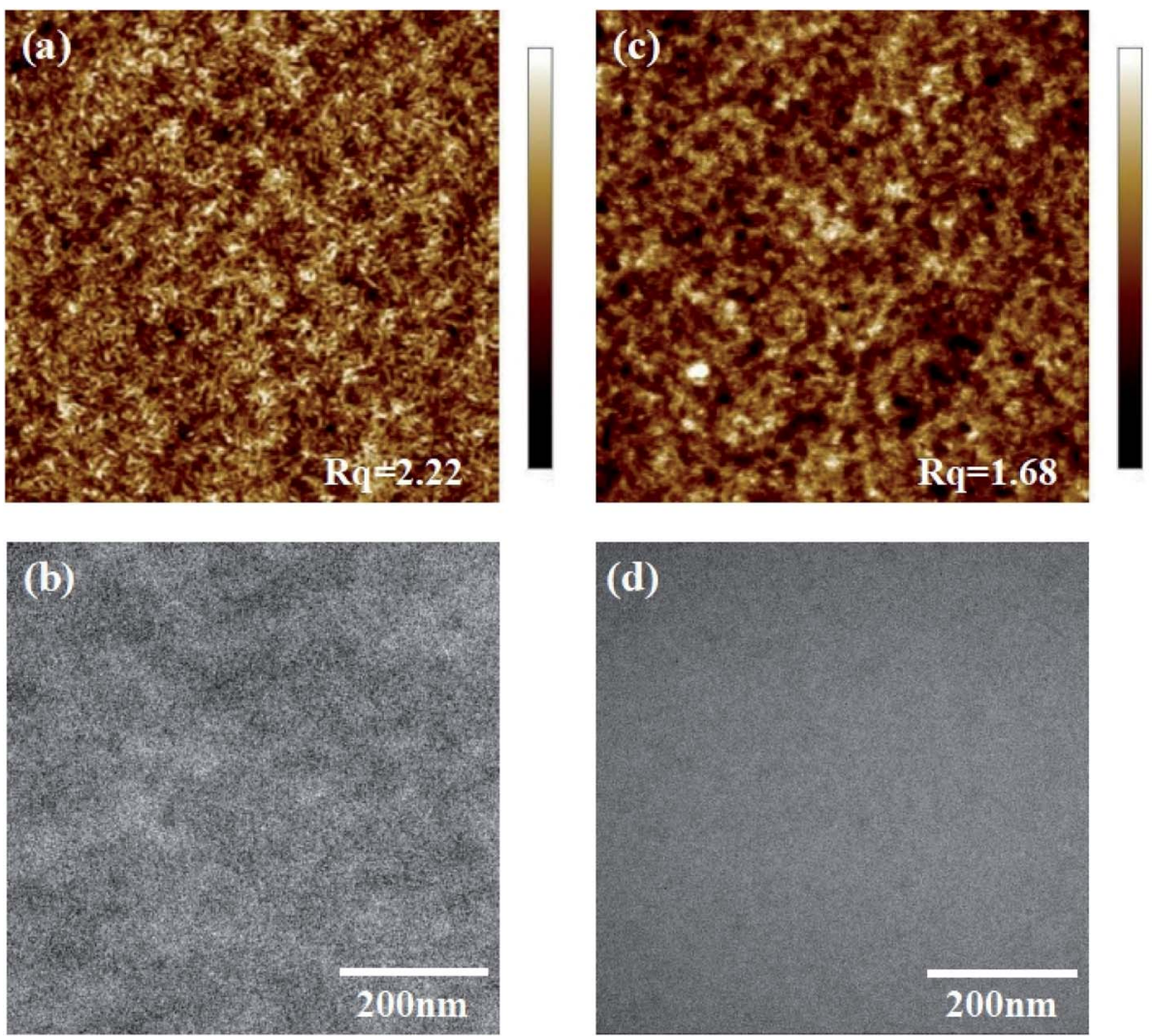

Fig. 4 The AFM and TEM images of PBDB-T:BZIC-2Br and PBDB-T:Y9-2Br blend films. (a and b) Height image (size $5 \times 5 \mu m^{2}$ ) of PBDB-T:BZIC2Br and PBDB-T:Y9-2Br; (c and d) TEM image $\left(2 \times 2 \mu \mathrm{m}^{2}\right)$ of PBDB-T:BZIC-2Br and PBDB-T:Y9-2Br. 


\section{Acknowledgements}

The work was supported by the National Natural Science Foundation of China (No. 21875286), the Hunan Intellectual Property Bureau (No. 2016e004), the Science and Technology Plan Key Project of Hunan Province (No. 2020GK2100) and the Natural Science Foundation of Hunan Province (No. 2020JJ4721).

\section{References}

1 S. X. Li, C. Z. Li, M. M. Shi and H. Z. Chen, New Phase for Organic Solar Cell Researches: Emergence of Y-Series Electron Acceptors and Their Perspectives, ACS Energy Lett., 2020, 5(5), 1554-1567.

2 L. L. Feng, J. Yuan, Z. Z. Zhang, H. J. Peng and Z. G. Zhang, Thieno[3,2-b]pyrrolo-Fused Pentacyclic Benzotriazole-Based Acceptor for Efficient Organic Photovoltaics, ACS Appl. Mater. Interfaces, 2017, 9, 31985-31992.

3 Y. Lin, J. Wang, Z. G. Zhang, H. Bai, Y. Li, D. Zhu and X. Zhan, An Electron Acceptor Challenging Fullerenes for Efficient Polymer Solar Cells, Adv. Mater., 2015, 7, 1170-1174.

4 H. Li, D. He, P. Mao, Y. Z. Wei, L. M. Ding and J. Z. Wang, Additive-Free Organic Solar Cells with Power Conversion Efficiency over 10\%, Adv. Energy Mater., 2017, 7(13), 1602663.

5 Q. S. Liu, Y. F. Jiang, K. Jin, J. Q. Qin, J. G. Xu and J. Xiong, 18\% Efficiency Organic Solar Cells, Sci. Bull., 2020, 65(4), 272-275.

6 C. Q. Yan, S. Barlow, Z. H. Wang, H. Yan, A. K.-Y. Jen, S. R. Marder and X. W. Zhan, Non-Fullerene Acceptors for Organic Solar Cells, Nat. Rev. Mater., 2018, 3, 18003.

7 G. Y. Zhang, J. B. Zhao, K. Jiang, J. Q. Zhang, Z. L. Zhu, J. Zhang, F. Huang and H. Yan, Non-fullerene Acceptor Molecules for Bulk Heterojunction Organic Solar Cells, Chem. Rev., 2018, 118, 3447-3507.

8 W. Zhao, S. Li, H. Yao, S. Zhang, Y. Zhang, B. Yang and J. H. Hou, Molecular Optimization Enables over $13 \%$ Efficiency in Organic Solar Cells, J. Am. Chem. Soc., 2017, 139, 7148-7151.

9 S. Li, L. Ye, W. Zhao, H. Yan, B. Yang, D. Liu, W. Li and J. H. Hou, A Wide Band Gap Polymer with a Deep Highest Occupied Molecular Orbital Level Enables 14.2\% Efficiency in Polymer Solar Cells, J. Am. Chem. Soc., 2018, 140, 71597167.

10 Y. Cui, H. Yao, L. Hong, T. Zhang, Y. Xu, K. Xian, B. Gao, J. Qin and J. Zhang, Achieving Over 15\% Efficiency in Organic Photovoltaic Cells Via Copolymer Design, Adv. Mater., 2019, 31, 1808356.

11 J. Wang, J. Zhang, Y. Xiao, T. Xiao, R. Zhu, C. Yan, Y. Fu and G. Lu, Effect of Isomerization on High-Performance Nonfullerene Electron Acceptors, J. Am. Chem. Soc., 2018, 140, 9140-9147.

12 B. Jia, J. Wang, Y. Wu, M. Zhang, Y. Jiang, Z. Tang and $\mathrm{X}$. Zhan, Enhancing the Performance of a Fused-Ring Electron Acceptor by Unidirectional Extension, J. Am. Chem. Soc., 2019, 141, 19023-19031.
13 B. Xiao, A. Tang, J. Zhang, A. Mahmood, Z. Wei and E. Zhou, Achievement of High $V_{\text {oc }}$ of $1.02 \mathrm{~V}$ for P3HT-Based Organic Solar Cell Using a Benzotriazole-Containing Non-Fullerene Acceptor, Adv. Energy Mater., 2017, 7, 1602269.

14 M.-F. Falzon, M. M. Wienk and R. A. J. Janssen, Designing Acceptor Polymers for Organic Photovoltaic Devices, $J$. Phys. Chem. C, 2011, 115, 3178-3187.

15 M. C. Gwinner, T. J. K. Brenner, J.-K. Lee, C. Newby, C. K. Ober, C. R. McNeill and H. Sirringhaus, Organic Field-Effect Transistors and Solar Cells Using Novel High Electron-Affinity Conjugated Copolymers Based on Alkylbenzotriazole and Benzothiadiazole, J. Mater. Chem., 2012, 22, 4436-4439.

16 K. Jiang, Q. Wei, J. Y. Lai, Z. Peng, H. K. Kim, J. Yuan, L. Ye, Y. P. Zou and H. Yan, Alkyl Chain Tuning of Small Molecule Acceptors for Efficient Organic Solar Cells, Joule, 2019, 3, 3020-3033.

17 Y. Cui, H. Yao, L. Hong, T. Zhang, Y. Tang, B. Lin, K. Xian, B. Gao, C. An, P. Bi and J. H. Hou, Organic Photovoltaic Cell with $17 \%$ Efficiency and Superior Processability, Natl. Sci. Rev., 2019, 7(7), 1239-1246.

18 J. Yuan, Y. Q. Zhang, L. Y. Zhou, G. Zhang, H. J. Peng and Y. P. Zou, Single-Junction Organic Solar Cell with over $15 \%$ Efficiency Using Fused-Ring Acceptor with ElectronDeficient Core, Joule, 2019, 3, 1140-1151.

19 P. Li, Z. X. Wang, C. P. Song and H. Y. Zhang, Rigid Fused pispacers in D-pi-A Type Molecules for Dye-sensitized Solar Cells: a Computational Investigation, J. Mater. Chem. C, 2017, 5, 11454-11465.

20 Q. Y. Wei, W. Liu, M. Leclerc, J. Yuan, H. G. Chen and Y. P. Zou, A-DA'D-A Non-Fullerene Acceptors for HighPerformance Organic Solar Cells, Sci. China: Chem., 2020, 63, 1352-1366.

21 Y. Zhang, J. Y. Zou, H. L. Yip, Y. Sun, J. A. Davies, K. S. Chen and O. Acton, Conjugated Polymers Based on C, Si and Nbridged Dithiophene and Thienopyrroledione Units: Synthesis, Field-effect Transistors and Bulk Heterojunction Polymer Solar Cells, J. Mater. Chem., 2011, 21, 3895-3902.

22 C. Dyer-Smith, I. A. Howard and C. Cabanetos, Interplay Between Side Chain Pattern, Polymer Aggregation, and Charge Carrier Dynamics in PBDTTPD:PCBM BulkHeterojunction Solar Cells, Adv. Energy Mater., 2015, 5, 1401778.

23 W. R. Liu, J. Y. Zhang, S. J. Xu and X. Z. Zhu, Efficient Organic Solar Cells Achieved at a Low Energy Loss, Sci. Bull., 2019, 64, 1144-1147.

24 S. Liu, J. Yuan, W. Deng, M. Luo, Y. Xie and Y. P. Zou, HighEfficiency Organic Solar Cells with Low Non-radiative Recombination Loss and Low Energetic Disorder, Nat. Photonics, 2020, 14, 300-305.

25 Z. Zhou, W. Liu, G. Zhou, M. Zhang, D. Qian, J. Zhang, S. Chen, S. Xu, C. Yang, F. Gao, H. Zhu and F. Liu, Subtle Molecular Tailoring Induces Significant Morphology Optimization Enabling over 16\% Efficiency Organic Solar Cells with Efficient Charge Generation, Adv. Mater., 2020, 32, 1906324. 
26 J. Yuan, Y. Zhang, L. Zhou, C. Zhang, T. K. Lau, G. Zhang, X. Lu, H. L. Yip, S. K. So and Y. Zou, Fused Benzothiadiazole: A Building Block for n-Type Organic Acceptor to Achieve High Performance Organic Solar Cells, Adv. Mater., 2019, 31, 1807577.

27 J. Yuan, T. Huang, P. Cheng, Y. Zou, H. Zhang, J. L. Yang, S. Y. Chang, F. Gao and Y. Yang, Enabling Low Voltage Losses and High Photocurrent in Fullerene-Free Organic Photovoltaics, Nat. Commun., 2019, 10, 570.

28 J. Yuan, Y. Zhang, L. Zhou, C. Zhang, T. K. Lau, G. Zhang, X. Lu, H. L. Yip, H. J. Peng and Y. P. Zou, Fused Benzothiadiazole: A Building Block for n-Type Organic Acceptor to Achieve High Performance Organic Solar Cells, Adv. Mater., 2019, 31, 1807577.

29 Z. Luo, R. Sun, C. Zhong, T. Liu, G. Zhang, Y. Zou, X. Jiao, J. Min and C. Yang, Altering Alkyl-Chains Branching Positions for Boosting the Performance of Small-Molecule Acceptors for Highly Efficient Nonfullerene Organic Solar Cells, Sci. China: Chem., 2020, 63, 361-369.

30 L. Hong, H. Yao, Z. Wu, Y. Cui, T. Zhang, Y. Xu, R. Yu, Q. Liao, B. Gao, K. Xian and J. H. Hou, Eco-Compatible Solvent-Processed Organic Photovoltaic Cells with over 16\% Efficiency, Adv. Mater., 2019, 31, 1903441.

31 T. J. Aldrich, M. Matta, W. Zhu, S. M. Swick, C. L. Stern, G. C. Schatz, A. Facchetti, F. S. Melkonyan and T. J. Marks, Fluorination Effects on Indacenodithienothiophene Acceptor Packing and Electronic Structure, End-Group Redistribution, and Solar Cell Photovoltaic Response, J. Am. Chem. Soc., 2019, 141, 3274-3287.

32 L. Zhan, S. Li, T. K. Lau, Y. Cui, X. Lu, M. Shi, C. Z. Li, H. Li, J. H. Hou and H. Chen, Over 17\% Efficiency Ternary Organic Solar Cells Enabled by Two Non-Fullerene Acceptors Working in an Alloy-Like Model, Energy Environ. Sci., 2020, 13, 635-645.

33 F. D. Eisner, M. Azzouzi, Z. Fei, X. Hou, T. D. Anthopoulos, T. J. S. Dennis, M. Heeney and J. Nelson, Hybridization of Local Exciton and Charge-Transfer States Reduces Nonradiative Voltage Losses in Organic Solar Cells, J. Am. Chem. Soc., 2019, 141, 6362-6374.

34 H. Yao, Y. Cui, R. Yu, B. Gao, H. Zhang and J. Hou, Design, Synthesis, and Photovoltaic Characterization of a Small Molecular Acceptor with an Ultra-Narrow Band Gap, Angew. Chem., Int. Ed., 2017, 56, 3045-3049.

35 M. L. Tang and Z. Bao, Halogenated Materials as Organic Semiconductors, Chem. Mater., 2011, 23, 446-455.

36 Y. Zhang, H. Yao, S. Zhang, Y. Qin, J. Zhang, L. Yang, W. Li, Z. Wei, F. Gao and J. Hou, Fluorination vs. Chlorination: a Case Study on High Performance Organic Photovoltaic Materials, Sci. China: Chem., 2018, 61, 1328-1337.

37 J. Qu, H. Chen, J. Zhou, H. Lai, T. Liu, P. Chao, D. Li, Z. Xie, F. He and Y. Ma, Chlorine Atom-Induced Molecular Interlocked Network in a Non-Fullerene Acceptor, ACS Appl. Mater. Interfaces, 2018, 10, 39992-40000.

38 F. Y. Cao, W. C. Huang, S. L. Chang and Y. J. Cheng, Angular Shaped 4,9-Dialkylnaphthodithiophene-Based Octacyclic Ladder Type Non-Fullerene Acceptors for High Efficiency
Ternary-Blend Organic Photovoltaics, Chem. Mater., 2018, 30, 4968-4977.

39 B. Kan, H. Feng, H. Yao, M. Chang, X. Wan, C. Li, J. Hou and Y. Chen, A Chlorinated Low-Bandgap Small-Molecule Acceptor for Organic Solar Cells with 14.1\% Efficiency and Low Energy Loss, Sci. China: Chem., 2018, 61, 1307-1313.

40 H. Chen, Z. Hu, H. Wang, L. Liu, P. Chao, J. Qu, W. Chen, A. Liu and F. He, A Chlorinated $\pi$-Conjugated Polymer Donor for Efficient Organic Solar Cells, Joule, 2018, 2, 1623-1634.

41 J. Qu, Q. Zhao, J. Zhou, H. Lai, T. Liu, D. Li, W. Chen, Z. Xie and F. He, Multiple Fused Ring-Based Near-Infrared Nonfullerene Acceptors with an Interpenetrated ChargeTransfer Network, Chem. Mater., 2019, 31, 1664-1671.

42 J. F. Qu, D. Li, H. Wang, J. D. Zhou, Z. Q. Xie and F. He, Bromination of the Small-Molecule Acceptor with Fixed Position for High-Performance Solar Cells, J. Mater. Chem. A, 2019, 31, 8044-8051.

43 H. Wang, T. Liu and J. D. Zhou, Bromination: An Alternative Strategy for Non-Fullerene Small Molecule Acceptors, J. Adv. Sci., 2020, 1903784-1903793.

44 F. Yang, C. Li, W. Lai, A. Zhang, H. Huang and W. Li, Halogenated Conjugated Molecules for Ambipolar FieldEffect Transistors and Non-Fullerene Organic Solar Cells, Mater. Chem. Front., 2017, 1, 1389-1395.

45 Y. Wang, Y. Zhang, N. Qiu, H. Feng, H. Gao, B. Kan, Y. Ma, C. Li, X. Wan and Y. Chen, A Halogenation Strategy for over $12 \%$ Efficiency Non-fullerene Organic Solar Cells, Adv. Energy Mater., 2018, 8(15), 1702870.

46 S. S. Wan, C. Chang, J. L. Wang, G. Z. Yuan, Q. Wu, M. Zhang and Y. Li, Effects of the Number of Bromine Substitution on Photovoltaic Efficiency and Energy Loss of Benzo[1,2- $b: 4,5$ $b^{\prime}$ ]-diselenophene-based Narrow-Bandgap Multibrominated Nonfullerene Acceptors, Sol. RRL, 2019, 3, 1800250.

47 J. Qu, D. Li, H. Wang, J. Zhou, N. Zheng, H. Lai, T. Liu, Z. Xie and F. He, Bromination of the Small-Molecule Acceptor with Fixed Position for High Performance Solar Cells, Chem. Mater., 2019, 31(19), 8044-8051.

48 H. Lai, Q. Zhao, Z. Chen, H. Chen, P. Chao, Y. Zhu, Y. Lang, N. Zhen, D. Mo, Y. Zhang and F. He, Trifluoromethylation Enables a 3D Interpenetrated Low Band-gap Acceptor for Efficient Organic Solar Cells, Joule, 2020, 4(3), 688-700.

49 C. Sun, S. Qin, R. Wang, S. Chen, F. Pan, B. Qiu, Z. Shang, L. Meng, C. Zhang, M. Xiao, C. Yang and Y. F. Li, High Efficiency Polymer Solar Cells with Efficient Hole Transfer at Zero Highest Occupied Molecular Orbital Offset Between Methylated Polymer Donor and Brominated Acceptor, J. Am. Chem. Soc., 2020, 142(3), 1465-1474.

50 H. Wang, T. Liu, T. Zhou, D. Mo, L. Han, H. Lai, H. Chen, N. Zheng, Y. Zhu, Z. Xie and F. He, Bromination: an Alternative Strategy for Non-fullerene Small Molecule Acceptors, Adv. Sci., 2020, 1903784.

51 S. Y. Chang, P. Cheng, G. Li and Y. Yang, Transparent Polymer Photovoltaics for Solar Energy Harvesting and Beyond, Joule, 2018, 2(6), 1039-1054.

52 H. Bai, Y. Wang, P. Cheng, Y. Li and D. Zhu, AcceptorDonor-Acceptor Small Molecules Based on 
Indacenodithiophene for Efficient Organic Solar Cells, ACS Appl. Mater. Interfaces, 2014, 6(11), 8426-8433.

53 W. Zhang, J. Smith, S. Watkins, R. Gysel, M. McGehee, A. Salleo, J. Kirkpatrick, S. Ashraf, T. Anthopoulos, M. Heeney and I. McCulloch, Indacenodithiophene Semiconducting Polymers for High-Performance, Air-Stable Transistors, J. Am. Chem. Soc., 2010, 132, 11437-11439.

54 Y. Li, X. Liu, F. P. Wu, Y. Zhou, Z. Q. Jiang, B. Song, Y. Xia and Z. G. Zhang, Non-fullerene Acceptor with Low Energy Loss and High External Quantum Efficiency: Towards High Performance Polymer Solar Cells, J. Mater. Chem. A, 2016, 16, 5890-5897.

55 S. Dai, F. Zhao, Q. Zhang, T. K. Lau, T. Li, K. Liu, Q. Ling, C. Wang, X. Lu, W. You and X. Zhan, Fused Nonacyclic
Electron Acceptors for Efficient Polymer Solar Cells, J. Am. Chem. Soc., 2017, 3, 1336-1343.

56 F. F. Cai, C. Zhu, J. Yuan, Z. Li, Y. F. Li and Y. P. Zou, Efficient Organic Solar Cells Based on a New "Y-series" Non-fullerene Acceptor with an Asymmetric Electron-Deficient-Core, Chem. Commun., 2020, 56, 4340-4343.

57 Y. Wang, Y. Zhang, N. Qiu, H. Feng, H. Gao, B. Kan, Y. Ma, C. Li, X. Wan and Y. Chen, A Halogenation Strategy for Over 12\% Efficiency Non-fullerene Organic Solar Cells, Adv. Energy Mater., 2018, 8(15), 1702870.

58 L. J. A. Koster, V. D. Mihailetchi, R. Ramaker and P. W. M. Blom, Light Intensity Dependence of Open-circuit Voltage of Polymer: Fullerene Solar Cells, Appl. Phys. Lett., 2005, 86, 123509. 